\title{
Age-Related Changes in Visceral Patterns Associated with Anxiety Disorders or Depression according to Traditional Chinese Medicine
}

\author{
Tetsuya Kondo $^{a}$ Kouichi Yoshimasu ${ }^{b}$ Shoji Tokunaga ${ }^{c}$ \\ Hideyo Sugahara $^{d}$ Yoshio Kanemitsu $^{e}$ Chiharu Kubo $^{f}$ \\ ${ }^{a}$ Kansai University of Health Sciences, Osaka, ${ }^{b}$ Department of Hygiene, School of Medicine, \\ Wakayama Medical University, Wakayama, ' $M e d i c a l$ Information Center, Kyushu University \\ Hospital, d Sugahara Tenjin Clinic, ${ }^{e}$ Department of Psychosomatic Medicine, Fukuoka Dental \\ College, and ${ }^{\mathrm{f}}$ International University of Health and Welfare, Fukuoka, Japan
}

Key Words

Anxiety · Depression · Patterns · Visceral pattern · Traditional Chinese medicine

\section{Abstract}

Background: Since, to our knowledge, there is no report on age-related changes in the patterns associated with anxiety and mood disorders, a large-size case-control study was conducted. Methods: A total of 914 new cases were assessed at a psychosomatic clinic of a tertiary medical care center. The severity of visceral patterns was analyzed according to the main symptoms described and a comprehensive questionnaire. Patterns of the liver (three), heart (four), and kidney (one) as well as dual deficiency of the heart and spleen were assessed. Results: In females under 40 years of age, liver qi depression and phlegm fire harassing the heart were associated with generalized anxiety disorder. Liver fire flaming upward was associated with all forms of anxiety disorders in this group. Phlegm fire harassing the heart was associated with depression and all forms of mood disorders in females over 40 years of age, while it was inversely associated in males over 40 years of age. Conclusion: The order of the traditional Chinese patterns resulting in anxiety or mood disorders was consistent with the development of liver qi depression into liver fire flaming upward or phlegm fire harassing the heart according to the traditional Chinese theory. The patterns associated with depression and all forms of mood disorders vary according to age and sex. 


\section{Introduction}

In a previous study, it was reported that up to $67.1 \%$ of patients with depression fail to respond to the first antidepressant that is prescribed [1]. Therefore, more than half of the patients with anxiety or depression consult complementary and alternative medicine practitioners for alternative treatments [2]. In fact, it was reported that a combined treatment of the antidepressant paroxetine and electroacupuncture was more effective at combating depressive disorders than paroxetine alone [3].

Depression has been reported to coexist with anxiety disorders such as generalized anxiety disorder and panic disorder [4]. This coexistence is called 'anxious depression'. A determinate trend is observed on the onset of the disorders [4]. The onset of anxiety disorders, especially generalized anxiety disorder, is often in the teens, while that of depression takes place later and frequently in the twenties and sometimes in the fifties [4]. This indicates that long-lasting anxiety leads to depression via the daily nerve strain. Changes with age also exist in the patterns according to traditional Chinese medicine. Some patterns are considered to result from other patterns. For example, intense heart fire results from liver qi depression. Different stages of sequential patterns are associated with anxiety disorders and depression according to traditional Chinese medicine. However, there are no reports on the age-related changes in the patterns of these disorders. Therefore, it would be useful to investigate the association between these disorders and the patterns.

Mood disorders, anxiety disorders, and insomnia have been treated with empirical therapy by using Kampo recipes for liver qi depression [5], intense heart fire [6], dual deficiency of the heart and spleen syndrome [7], yin deficiency [7], phlegm fire harassing the heart [8], and phlegm turbidity [8]. When associated with these disorders, yin deficiency is categorized into three forms: liver, heart, and kidney yin deficiency.

In the present study, the associations between diagnoses of anxiety and mood disorders according to the Diagnostic and Statistical Manual of Mental Disorders, 4th edition (DSM-IV) [9] and these nine patterns were assessed.

\section{Materials and Methods}

\section{Subjects}

This study included 914 outpatients that visited for the first time the Department of Psychosomatic Medicine at the Kyushu University Hospital (a tertiary medical care center) between June 2000 and March 2001. Approximately half of them were walk-in patients and the remaining were referrals. All patients provided written informed consent to participate in the study. The Research Ethics Committee of the Graduate School of Medical Sciences, Kyushu University, approved the protocol of the present survey.

\section{Diagnoses}

After pre-examination by a physician specialized in psychosomatic medicine, the patients were assigned to the attending physicians and underwent a thorough checkup. Then, a diagnosis was made by the attending physician, according to the DSM-IV. All physicians were also familiar with clinical psychiatry, since they routinely examine patients with mood, anxiety, somatoform, and eating disorders.

\section{Identification of Visceral Patterns}

It was investigated whether each symptom corresponding to liver qi depression, liver fire flaming upward, dual deficiency of the heart and spleen, intense heart fire, phlegm turbidity, phlegm fire harassing the heart, heart yin deficiency, liver yin deficiency or kidney yin deficiency was present in their chief complaints or answers to questionnaires.

Since there are no reports on a systematic scoring system according to traditional Chinese medicine, the scoring for the patterns was conducted provisionally as follows. At the preliminary examination, all patients 
were asked to describe their main symptoms (up to three complaints). They were also asked to complete the Self-Rating Depression Scale (SDS) and the Kyudai Medical Index (KMI) questionnaire. The KMI is a modification of the Cornell Medical Index-Health Questionnaire [10] and consists of 103 yes-or-no questions, which include two comprehensive questions as well as questions on past history (two), family history (two), psychiatric symptoms (50), and somatic symptoms (47). The symptom that was described as a chief complaint was considered as one point for each visceral pattern. Symptoms that were not described as a chief complaint, but were mentioned in the KMI, were regarded as half a point for each visceral pattern, since these symptoms can be regarded as relatively minor. The correspondence between the symptoms described as chief complaints or featured in the KMI and each visceral pattern is shown in Appendix 1. The KMI question numbers of the symptoms included in the KMI are also listed. The visceral pattern values were expressed as percentages of the full marks. The World Health Organization (WHO) International Standard terminologies on traditional medicine in the Western Pacific region were referred to for all traditional Chinese medicine terminologies [11].

Statistics

Subjects with organic neurological disorders, endocrine disorders, neoplasms, vascular disorders, infections, degenerative diseases or traumatism were excluded from the analyses. If a diagnosis could not be identified, the subject was excluded from the study. Other exclusion criteria included failure of the patient to complete the KMI (more than 21 questions unanswered) or to describe the main complaints. Thus, a total of 579 subjects were included.

All analyses were performed using Stata version 10.1 (Stata Corp., College Station, Tex., USA). Each pattern score was divided equally into lowest, lower, higher, and highest groups in an ascending order. The subjects were divided into a younger ( $<40$ years) female group, an older ( $\geq 40$ years) female group, a younger ( $<40$ years) male group, and an older ( $\geq 40$ years) male group. Multiple logistic regression analyses were performed to estimate the association between the prevalence of occidental diagnoses and the visceral pattern scores. If any case numbers were zero, logistic regression analyses were performed without adjusting for age. A two-sided p value of 0.05 was considered to be statistically significant.

\section{Results}

\section{Demographic and Occidental Status}

The demographic data of the subjects and the prevalence of the diagnoses are given in table 1. Continuous variables are shown as mean \pm standard deviation. The numbers and percentages of patients for each disorder are presented as well. The mean age of the subjects was 36.4 years, with no significant sex differences. The prevalence of mood, anxiety, eating, and somatoform disorders was 54.2, 19.9, 10.9, and 7.6\%, respectively. Owing to overlapping, the total number of patients with subclassified diseases did not equal the number in each corresponding major division.

The average SDS scores in the younger and older females were more than 50 , which indicates an apparent depressive state [12], while those of the male subjects were less than 50. In all groups, the average STAI-I scores were more than 42, which indicates an apparent state of anxiety [13]. In addition, in all groups, the average STAI-II scores were more than 44 or 45 , which indicates an apparent anxiety trait [13].

It has been reported that the KMI is able to distinguish healthy controls from patients with neurotic disorders and those with psychosomatic disorders [10]. In this report, the category B question scores were the highest in the subjects with neurosis. The scores in the subjects with psychosomatic disorders were slightly lower than in those with neurosis and considerably higher than in normal subjects [10]. In the present study, the average point score for category B questions (i.e., questions assessing psychiatric symptoms) in males under 40 years of age was 23.1 of 50, which was higher than the average reported previously in normal subjects ( 9.5 of 50 ) and in subjects with psychosomatic disorders (18.8 of 
Table 1. Demographic data on the subjects

\begin{tabular}{|c|c|c|c|c|c|}
\hline & \multicolumn{2}{|c|}{ Female $(\mathrm{n}=364)$} & \multicolumn{2}{|c|}{ Male $(n=215)$} & \multirow{2}{*}{$\begin{array}{l}\text { Total } \\
(n=579)\end{array}$} \\
\hline & $\begin{array}{l}<40 \text { years } \\
(\mathrm{n}=229)\end{array}$ & $\begin{array}{l}>40 \text { years } \\
(\mathrm{n}=135)\end{array}$ & $\begin{array}{l}<40 \text { years } \\
(\mathrm{n}=138)\end{array}$ & $\begin{array}{l}>40 \text { years } \\
(\mathrm{n}=77)\end{array}$ & \\
\hline Age, years & $25.0 \pm 6.7$ & $55.9 \pm 10.1$ & $25.5 \pm 7.5$ & $55.4 \pm 10.9$ & $36.4 \pm 16.9$ \\
\hline SDS points & $52.8 \pm 9.8$ & $50.5 \pm 10.4$ & $48.7 \pm 9.8$ & $47.6 \pm 11.3$ & $50.6 \pm 10.3$ \\
\hline STAI-I points & $55.6 \pm 11.4$ & $55 \pm 10.9$ & $53.9 \pm 11.7$ & $53.1 \pm 12.1$ & $54.8 \pm 11.5$ \\
\hline STAI-II points & $57.5 \pm 12$ & $53.4 \pm 10.9$ & $54.6 \pm 11.3$ & $52.7 \pm 14$ & $55.3 \pm 12.0$ \\
\hline KMI category B questions points & $24.5 \pm 9.7$ & $21.8 \pm 11.0$ & $23.1 \pm 10.5$ & $22.7 \pm 10.9$ & $23.3 \pm 10.4$ \\
\hline $\mathrm{KMI}$ category $\mathrm{C}$ questions points & $17.3 \pm 8.1$ & $15.9 \pm 8.7$ & $14.3 \pm 7.5$ & $16.0 \pm 7.7$ & $16.1 \pm 8.2$ \\
\hline Mood disorders & $112(48.9)$ & $93(68.9)$ & $68(49.3)$ & $41(53.2)$ & $314(54.2)$ \\
\hline Major depression & 85 (41.7) & $73(62.9)$ & $45(38.8)$ & $34(48.6)$ & $237(46.8)$ \\
\hline Dysthymic disorder & $10(4.4)$ & $13(9.6)$ & $4(2.9)$ & $4(5.2)$ & $31(5.4)$ \\
\hline Depressive disorder not otherwise specified & $26(11.4)$ & $16(11.9)$ & $22(15.9)$ & $7(9.1)$ & $71(12.3)$ \\
\hline Anxiety disorders & $46(20.1)$ & $25(18.5)$ & $34(24.6)$ & $10(13.0)$ & $115(19.9)$ \\
\hline Generalized anxiety disorder & $15(6.6)$ & $20(14.8)$ & $15(10.9)$ & $7(9.1)$ & $57(9.8)$ \\
\hline Specific phobia & $3(1.3)$ & 0 & $1(0.7)$ & $1(1.3)$ & $5(0.9)$ \\
\hline Panic disorder & $19(8.3)$ & $5(3.7)$ & $8(5.8)$ & $3(3.9)$ & $35(6.0)$ \\
\hline Posttraumatic stress disorder & $1(0.4)$ & 0 & $2(1.4)$ & 0 & $3(0.5)$ \\
\hline Social anxiety disorder & $4(1.7)$ & 0 & $8(5.8)$ & 0 & $12(2.1)$ \\
\hline Obsessive-compulsive disorder & $5(2.2)$ & 0 & $1(0.7)$ & 0 & $6(1.0)$ \\
\hline Eating disorders & $56(24.5)$ & $1(0.7)$ & $5(3.6)$ & $1(1.3)$ & $63(10.9)$ \\
\hline Anorexia nervosa & $16(7.0)$ & $1(0.7)$ & $2(1.4)$ & $1(1.3)$ & $20(3.5)$ \\
\hline Bulimia nervosa & $27(11.8)$ & 0 & $1(0.7)$ & 0 & $28(4.8)$ \\
\hline Eating disorder not otherwise specified & $13(5.7)$ & 0 & $2(1.4)$ & 0 & $15(2.6)$ \\
\hline Somatoform disorders & $13(5.7)$ & $15(11.1)$ & $6(4.3)$ & $10(13.0)$ & $44(7.6)$ \\
\hline Conversion disorder & $2(0.9)$ & $2(1.5)$ & 0 & 0 & $4(0.7)$ \\
\hline Hypochondriasis & 0 & $3(2.2)$ & $1(0.7)$ & $2(2.6)$ & $6(1.0)$ \\
\hline Somatization disorder & 0 & $1(0.7)$ & 0 & 0 & $1(0.2)$ \\
\hline Pain disorder & $9(3.9)$ & $6(4.4)$ & $3(2.2)$ & $7(9.1)$ & $25(4.3)$ \\
\hline Somatoform disorder not otherwise specified & $2(0.9)$ & $2(1.5)$ & $1(0.7)$ & $1(1.3)$ & $6(1.0)$ \\
\hline Irritable bowel syndrome & $15(6.6)$ & $9(6.7)$ & $8(5.8)$ & $6(7.8)$ & $38(6.6)$ \\
\hline Functional dyspepsia & $15(6.6)$ & 0 & $8(5.8)$ & $1(1.3)$ & $24(4.1)$ \\
\hline Primary insomnia & $5(2.2)$ & $2(1.5)$ & $6(4.3)$ & $7(9.1)$ & $20(3.5)$ \\
\hline Hypertension & $4(1.7)$ & $4(3.0)$ & $3(2.2)$ & $2(2.6)$ & $13(2.2)$ \\
\hline Hyperventilation syndrome & $8(3.5)$ & $2(1.5)$ & $2(1.4)$ & 0 & $12(2.1)$ \\
\hline Bronchial asthma & $5(2.2)$ & $3(2.2)$ & $1(0.7)$ & 0 & $9(1.6)$ \\
\hline Dystonia & $2(0.9)$ & $4(3.0)$ & $1(0.7)$ & $1(1.3)$ & $8(1.4)$ \\
\hline Schizophrenia & $2(0.9)$ & $2(1.5)$ & 0 & $4(5.2)$ & $8(1.4)$ \\
\hline Chronic fatigue syndrome & 0 & $2(1.5)$ & $1(0.7)$ & 0 & $3(0.5)$ \\
\hline Dissociative disorder & $2(0.9)$ & 0 & 0 & 0 & $2(0.3)$ \\
\hline
\end{tabular}

Values in parentheses are percentages.

50 ) but lower than in those with neurosis (24.4 of 50). The average point score for category C questions in this group was 14.3 , which was lower than those of patients with psychosomatic disorders (14.8) and neurosis (23.1 of 50) [10]. Thus, this group was regarded as psychosomatic as well as neurotic. However, in the other three groups, the average point scores for category B questions were all higher than those of psychosomatic disorders and close to those of neurosis. The average point scores for category $\mathrm{C}$ questions were higher than those of neurosis. Thus, these groups were regarded as neurotic rather than psychosomatic. 
Kondo et al.: Age-Related Changes in Visceral Patterns Associated with Anxiety

Table 2. Visceral pattern scores

\begin{tabular}{|c|c|c|c|c|c|c|c|}
\hline & \multicolumn{3}{|c|}{ Female $(n=364)$} & \multicolumn{3}{|c|}{ Male $(n=215)$} & \multirow{2}{*}{$\begin{array}{l}\text { Total } \\
(n=579)\end{array}$} \\
\hline & $\begin{array}{l}<40 \text { years } \\
(n=229)\end{array}$ & $\begin{array}{l}>40 \text { years } \\
(n=135)\end{array}$ & $\begin{array}{l}\text { difference } \\
\text { (over - below) }\end{array}$ & $\begin{array}{l}<40 \text { years } \\
(n=138)\end{array}$ & $\begin{array}{l}>40 \text { years } \\
(n=77)\end{array}$ & $\begin{array}{l}\text { difference } \\
\text { (over-below) }\end{array}$ & \\
\hline Liver qi depression & $11.9 \pm 6.0$ & $11.0 \pm 5.9$ & -0.9 & $11.9 \pm 6.4$ & $11.1 \pm 5.3$ & -0.8 & $11.6 \pm 6.0$ \\
\hline Liver fire flaming upward & $17.1 \pm 9.1$ & $16.0 \pm 9.2$ & -1.1 & $17.3 \pm 9.8$ & $17.2 \pm 9.2$ & -0.1 & $16.9 \pm 9.3$ \\
\hline Intense heart fire & $14.1 \pm 9.2$ & $13.6 \pm 8.9$ & -0.5 & $13.6 \pm 8.6$ & $13.9 \pm 8.5$ & +0.3 & $13.8 \pm 8.9$ \\
\hline Phlegm fire harassing the heart & $14.4 \pm 7.7$ & $13.6 \pm 7.3$ & -0.8 & $14.7 \pm 7.1$ & $14.4 \pm 8.1$ & -0.3 & $14.3 \pm 7.5$ \\
\hline Phlegm turbidity & $15.1 \pm 10.9$ & $13.9 \pm 9.5$ & -1.2 & $16.6 \pm 11.3$ & $14.6 \pm 10.7$ & -2.0 & $15.1 \pm 10.7$ \\
\hline Dual deficiency of the heart and spleen & $21.5 \pm 9.7$ & $21.2 \pm 11.0$ & -0.3 & $21.7 \pm 9.8$ & $21.2 \pm 10.7$ & -0.5 & $21.4 \pm 10.2$ \\
\hline Liver yin deficiency & $14.3 \pm 7.3$ & $14.0 \pm 7.5$ & -0.3 & $13.7 \pm 7.5$ & $13.2 \pm 7.0$ & -0.5 & $14.0 \pm 7.4$ \\
\hline Heart yin deficiency & $17.4 \pm 8.8$ & $17.0 \pm 8.9$ & -0.4 & $16.9 \pm 9.3$ & $17.2 \pm 9.1$ & +0.3 & $17.2 \pm 9.0$ \\
\hline Kidney yin deficiency & $11.4 \pm 6.2$ & $11.5 \pm 6.4$ & +0.1 & $11.5 \pm 7.0$ & $11.0 \pm 5.8$ & -0.5 & $11.4 \pm 6.4$ \\
\hline
\end{tabular}

Table 3. Associations between generalized anxiety disorder and visceral patterns

\begin{tabular}{|c|c|c|c|c|c|c|c|c|}
\hline \multirow{2}{*}{$\begin{array}{l}\text { Pattern/ } \\
\text { extent }\end{array}$} & \multicolumn{2}{|l|}{ Younger female group } & \multicolumn{2}{|l|}{ Older female group } & \multicolumn{2}{|l|}{ Younger male group } & \multicolumn{2}{|c|}{ Older male group } \\
\hline & OR $(95 \% \mathrm{CI})$ & $\begin{array}{l}\mathrm{p} \\
\text { value }\end{array}$ & OR $(95 \% \mathrm{CI})$ & $\begin{array}{l}\mathrm{p} \\
\text { value }\end{array}$ & OR $(95 \% \mathrm{CI})$ & $\begin{array}{l}\mathrm{p} \\
\text { value }\end{array}$ & OR & $\begin{array}{l}\mathrm{p} \\
\text { value }\end{array}$ \\
\hline \multicolumn{9}{|c|}{ Liver qi depression } \\
\hline Lower & $79.49(0.81-7,777.62)$ & 0.06 & $1.22(0.15-9.69)$ & 0.85 & $0.25(0.00-14.05)$ & 0.50 & - & 1.00 \\
\hline Higher & $440.53(1.41-138,132.32)$ & $<0.05$ & $1.64(0.13-20.22)$ & 0.70 & $0.62(0.00-411.79)$ & 0.89 & - & 1.00 \\
\hline Highest & $10.79(0.10-1,118.94)$ & 0.32 & $3.37(0.17-68.80)$ & 0.43 & - & 0.99 & - & 1.00 \\
\hline \multicolumn{9}{|c|}{ Liver fire flaming upward } \\
\hline Lower & $1.65(0.07-36.46)$ & 0.75 & $0.06(0.00-1.22)$ & 0.07 & $1.29(0.02-95.16)$ & 0.91 & - & 1.00 \\
\hline Higher & $0.00(0.00-0.83)$ & $<0.05$ & $0.09(0.00-3.71)$ & 0.20 & $0.00(0.00-251.29)$ & 0.19 & - & 1.00 \\
\hline Highest & $0.00(0.00-3.69)$ & 0.11 & $2.68(0.04-202.79)$ & 0.65 & - & 0.98 & - & 1.00 \\
\hline \multicolumn{9}{|c|}{ Dual deficiency of the heart and spleen } \\
\hline Lower & $0.34(0.01-9.96)$ & 0.53 & $1.55(0.17-13.77)$ & 0.69 & $0.30(0.01-17.82)$ & 0.56 & - & 1.00 \\
\hline Higher & $27.70(0.42-1,823.80)$ & 0.12 & $2.09(0.18-24.71)$ & 0.56 & $0.00(0.00-4.22)$ & 0.08 & - & 1.00 \\
\hline Highest & $0.65(0.01-41.05)$ & 0.84 & $0.23(0.01-7.57)$ & 0.41 & $0.18(0.00-149.21)$ & 0.61 & - & 1.00 \\
\hline \multicolumn{9}{|c|}{ Intense heart fire } \\
\hline Lower & $156.03(0.10-246,496.81)$ & 0.18 & $3.00(0.18-49.16)$ & 0.44 & $0.01(0.00-1.75)$ & 0.08 & - & 1.00 \\
\hline Higher & $2.01(0.00-3,353.92)$ & 0.85 & $12.76(0.19-838.00)$ & 0.23 & $6.61(0.00-40,623.97)$ & 0.67 & - & 1.00 \\
\hline Highest & $9.15(0.00-26,828.83)$ & 0.59 & $0.96(0.02-50.22)$ & 0.99 & - & 0.99 & - & 1.00 \\
\hline \multicolumn{9}{|c|}{ Phlegm turbidity } \\
\hline Lower & $0.03(0.00-0.61)$ & $<0.05$ & $2.07(0.30-14.20)$ & 0.46 & - & 0.97 & - & 1.00 \\
\hline Higher & $0.04(0.00-1.00)$ & $<0.05$ & $0.21(0.02-2.95)$ & 0.25 & $6,820,513.05(0.01-5,575,000,000,000,000.00)$ & 0.13 & - & 1.00 \\
\hline Highest & $0.01(0.00-0.50)$ & $<0.05$ & $0.87(0.03-30.01)$ & 0.94 & $0.05(0.00-52.67)$ & 0.39 & - & 0.99 \\
\hline \multicolumn{9}{|c|}{ Phlegm fire harassing the heart } \\
\hline Lower & $12.67(0.18-872.52)$ & 0.24 & $1.32(0.15-11.83)$ & 0.80 & $0.03(0.00-4.74)$ & 0.17 & - & 1.00 \\
\hline Higher & $58.55(0.16-21,123.22)$ & 0.18 & $2.48(0.07-93.45)$ & 0.62 & $41.48(0.02-100,004.93)$ & 0.35 & - & 1.00 \\
\hline Highest & $159.16(0.46-55,448.38)$ & 0.09 & $2.10(0.02-201.05)$ & 0.75 & - & 0.98 & - & 1.00 \\
\hline \multicolumn{9}{|c|}{ Liver yin deficiency } \\
\hline Lower & $0.30(0.01-7.34)$ & 0.46 & $3.50(0.39-30.98)$ & 0.26 & $11.00(0.11-1,129.84)$ & 0.31 & - & 1.00 \\
\hline Higher & $1.00(0.02-68.58)$ & 1.00 & $2.50(0.06-107.72)$ & 0.63 & $3.03(0.00-15,964.68)$ & 0.80 & - & 1.00 \\
\hline Highest & $0.10(0.00-5.60)$ & 0.26 & $1.93(0.07-56.11)$ & 0.70 & - & 0.99 & - & 1.00 \\
\hline \multicolumn{9}{|c|}{ Heart yin deficiency } \\
\hline Lower & $0.21(0.00-13.49)$ & 0.47 & $0.26(0.02-3.28)$ & 0.30 & $4,267.46(0.25-71,806,497.62)$ & 0.09 & - & 1.00 \\
\hline Higher & $0.41(0.00-43.14)$ & 0.71 & $0.38(0.02-7.82)$ & 0.53 & $31,084.63(0.01-84,742,590,131.23)$ & 0.17 & - & 1.00 \\
\hline Highest & $0.10(0.00-19.50)$ & 0.39 & $0.02(0.00-0.70)$ & $<0.05$ & - & 0.99 & - & 1.00 \\
\hline \multicolumn{9}{|c|}{ Kidney yin deficiency } \\
\hline Lower & $0.24(0.01-6.79)$ & 0.40 & $0.62(0.08-4.89)$ & 0.65 & $0.56(0.00-212.70)$ & 0.85 & - & 1.00 \\
\hline Higher & $0.18(0.00-8.83)$ & 0.39 & $0.94(0.05-19.27)$ & 0.97 & $63.43(0.09-47,043.43)$ & 0.22 & - & $>0.9999$ \\
\hline
\end{tabular}


Kondo et al.: Age-Related Changes in Visceral Patterns Associated with Anxiety

Disorders or Depression according to Traditional Chinese Medicine

Table 4. Associations between all forms of anxiety disorders and visceral patterns

\begin{tabular}{|c|c|c|c|c|c|c|c|c|}
\hline \multirow[t]{2}{*}{ Pattern/extent } & \multicolumn{2}{|c|}{ Younger female group } & \multicolumn{2}{|l|}{ Older female group } & \multicolumn{2}{|l|}{ Younger male group } & \multicolumn{2}{|c|}{ Older male group } \\
\hline & OR $(95 \% \mathrm{CI})$ & $\mathrm{p}$ value & OR $(95 \% \mathrm{CI})$ & p value & OR $(95 \% \mathrm{CI})$ & $\mathrm{p}$ value & OR & p value \\
\hline \multicolumn{9}{|c|}{ Liver qi depression } \\
\hline Lower & $3.13(0.83-11.79)$ & 0.09 & $1.91(0.29-12.66)$ & 0.50 & $3.67(0.70-19.39)$ & 0.13 & - & 1.00 \\
\hline Higher & $3.47(0.83-14.49)$ & 0.09 & $2.11(0.19-23.42)$ & 0.54 & $2.02(0.35-11.69)$ & 0.43 & - & 1.00 \\
\hline Highest & $2.76(0.57-13.29)$ & 0.21 & $6.06(0.31-118.20)$ & 0.23 & $1.09(0.09-13.11)$ & 0.95 & - & 1.00 \\
\hline \multicolumn{9}{|c|}{ Liver fire flaming upward } \\
\hline Lower & $2.01(0.57-7.07)$ & 0.28 & $0.06(0.00-0.92)$ & $<0.05$ & $1.66(0.31-8.92)$ & 0.56 & - & 1.00 \\
\hline Higher & $1.78(0.29-11.11)$ & 0.54 & $0.29(0.01-9.49)$ & 0.49 & $4.03(0.36-44.92)$ & 0.26 & - & 1.00 \\
\hline Highest & $9.07(1.22-67.51)$ & $<0.05$ & $2.80(0.05-148.65)$ & 0.61 & $1.12(0.06-22.49)$ & 0.94 & - & 0.99 \\
\hline \multicolumn{9}{|c|}{ Dual deficiency of the heart and spleen } \\
\hline Lower & $0.61(0.17-2.16)$ & 0.44 & $5.17(0.58-45.85)$ & 0.14 & $1.43(0.25-8.18)$ & 0.69 & - & 1.00 \\
\hline Higher & $2.29(0.47-11.28)$ & 0.31 & $2.69(0.24-30.08)$ & 0.42 & $0.97(0.16-5.71)$ & 0.97 & - & 0.99 \\
\hline Highest & $0.90(0.15-5.47)$ & 0.91 & $0.17(0.01-5.12)$ & 0.31 & $1.14(0.15-8.79)$ & 0.90 & - & 1.00 \\
\hline \multicolumn{9}{|l|}{ Intense heart fire } \\
\hline Lower & $1.00(0.26-3.86)$ & 1.00 & $1.10(0.09-12.83)$ & 0.94 & $0.37(0.07-2.05)$ & 0.26 & - & 1.00 \\
\hline Higher & $0.59(0.08-4.70)$ & 0.62 & $4.69(0.08-289.84)$ & 0.46 & $0.60(0.05-7.50)$ & 0.69 & - & 1.00 \\
\hline Highest & $0.41(0.05-3.18)$ & 0.40 & $0.48(0.01-21.65)$ & 0.71 & $0.55(0.03-11.28)$ & 0.70 & - & 1.00 \\
\hline \multicolumn{9}{|l|}{ Phlegm turbidity } \\
\hline Lower & $0.52(0.18-1.48)$ & 0.22 & $4.53(0.63-32.67)$ & 0.13 & $1.49(0.33-6.72)$ & 0.61 & - & 1.00 \\
\hline Higher & $0.49(0.12-2.06)$ & 0.33 & $0.22(0.02-2.57)$ & 0.23 & $0.42(0.08-2.33)$ & 0.32 & - & 1.00 \\
\hline Highest & $0.25(0.05-1.17)$ & 0.08 & $0.45(0.03-7.61)$ & 0.58 & $2.25(0.27-18.68)$ & 0.45 & - & 0.99 \\
\hline \multicolumn{9}{|c|}{ Phlegm fire harassing the heart } \\
\hline Lower & $1.85(0.46-7.37)$ & 0.39 & $1.18(0.16-8.82)$ & 0.87 & $0.18(0.03-0.94)$ & $<0.05$ & - & 1.00 \\
\hline Higher & $1.28(0.22-7.48)$ & 0.79 & $2.01(0.06-70.31)$ & 0.70 & $0.54(0.05-5.60)$ & 0.61 & - & 1.00 \\
\hline Highest & $2.31(0.33-16.38)$ & 0.40 & $5.24(0.05-506.78)$ & 0.48 & $1.86(0.07-50.88)$ & 0.71 & - & 1.00 \\
\hline \multicolumn{9}{|c|}{ Liver yin deficiency } \\
\hline Lower & $0.40(0.10-1.57)$ & 0.19 & $2.56(0.35-18.91)$ & 0.36 & $2.51(0.48-13.04)$ & 0.28 & - & 1.00 \\
\hline Higher & $0.14(0.02-0.91)$ & $<0.05$ & $0.59(0.02-16.27)$ & 0.76 & $1.49(0.15-14.86)$ & 0.74 & - & 1.00 \\
\hline Highest & $0.09(0.01-0.66)$ & $<0.05$ & $0.28(0.01-6.77)$ & 0.44 & $0.20(0.01-8.44)$ & 0.40 & - & 1.00 \\
\hline \multicolumn{9}{|c|}{ Heart yin deficiency } \\
\hline Lower & $1.38(0.32-5.91)$ & 0.67 & $0.42(0.04-4.29)$ & 0.46 & $0.92(0.15-5.58)$ & 0.93 & - & 1.00 \\
\hline Higher & $0.78(0.13-4.56)$ & 0.78 & $0.61(0.04-10.62)$ & 0.74 & $0.87(0.07-10.84)$ & 0.92 & - & 1.00 \\
\hline Highest & $0.54(0.07-4.00)$ & 0.54 & $0.05(0.00-1.45)$ & 0.08 & $0.97(0.04-21.61)$ & 0.98 & - & 0.99 \\
\hline \multicolumn{9}{|c|}{ Kidney yin deficiency } \\
\hline Lower & $0.56(0.15-2.05)$ & 0.38 & $0.61(0.09-4.25)$ & 0.62 & $1.02(0.20-5.23)$ & 0.98 & - & 1.00 \\
\hline Higher & $0.69(0.16-3.00)$ & 0.63 & $2.02(0.14-29.28)$ & 0.61 & $1.57(0.22-11.33)$ & 0.65 & - & 1.00 \\
\hline Highest & $1.20(0.16-9.15)$ & 0.86 & - & 0.97 & $8.37(0.55-126.63)$ & 0.13 & - & 1.00 \\
\hline
\end{tabular}

Visceral Patterns

The scores for each visceral pattern are given in table 2 . Analysis of variance revealed no differences between the groups in any pattern.

\section{Association between Anxiety Disorders and Visceral Patterns}

Associations between generalized anxiety disorder and visceral patterns are shown in table 3. The adjusted odds ratio (OR) and its 95\% confidence interval (CI) are presented. Liver qi depression was significantly associated with generalized anxiety disorder in the younger female group. Phlegm turbidity was inversely associated with this disorder. No significant association between generalized anxiety disorder and the patterns was observed in the other groups. Although not shown, no pattern was significantly associated with panic disorder in any group.

Associations between all forms of anxiety disorders and visceral patterns were also investigated and are shown in table 4. Liver fire flaming upward was significantly associated 
Kondo et al.: Age-Related Changes in Visceral Patterns Associated with Anxiety

Table 5. Associations between major depression and visceral patterns

\begin{tabular}{|c|c|c|c|c|c|c|c|c|}
\hline \multirow[t]{2}{*}{ Pattern/extent } & \multicolumn{2}{|c|}{ Younger female group } & \multicolumn{2}{|l|}{ Older female group } & \multicolumn{2}{|l|}{ Younger male group } & \multicolumn{2}{|l|}{ Older male group } \\
\hline & OR $(95 \% \mathrm{CI})$ & $\mathrm{p}$ value & OR $(95 \% \mathrm{CI})$ & $\mathrm{p}$ value & OR $(95 \% \mathrm{CI})$ & $\mathrm{p}$ value & OR $(95 \% \mathrm{CI})$ & $\mathrm{p}$ value \\
\hline \multicolumn{9}{|c|}{ Liver qi depression } \\
\hline Lower & $0.80(0.29-2.19)$ & 0.66 & $0.33(0.07-1.56)$ & 0.16 & $0.79(0.18-3.38)$ & 0.75 & $1.17(0.13-10.22)$ & 0.89 \\
\hline Higher & $1.20(0.41-3.56)$ & 0.74 & $0.11(0.02-0.80)$ & $<0.05$ & $1.15(0.23-5.68)$ & 0.86 & $1.41(0.09-21.78)$ & 0.80 \\
\hline Highest & $1.84(0.49-6.91)$ & 0.37 & $0.21(0.03-1.55)$ & 0.12 & $1.05(0.14-8.00)$ & 0.96 & $12.88(0.52-321.13)$ & 0.12 \\
\hline \multicolumn{9}{|c|}{ Liver fire flaming upward } \\
\hline Lower & $0.67(0.25-1.78)$ & 0.42 & $0.67(0.07-6.16)$ & 0.72 & $1.99(0.42-9.53)$ & 0.39 & $0.65(0.04-11.57)$ & 0.77 \\
\hline Higher & $0.67(0.16-2.76)$ & 0.58 & $0.66(0.05-8.79)$ & 0.75 & $0.64(0.08-5.39)$ & 0.68 & $0.29(0.01-8.50)$ & 0.48 \\
\hline Highest & $0.55(0.13-2.41)$ & 0.43 & $0.35(0.02-6.62)$ & 0.49 & $0.67(0.04-10.83)$ & 0.78 & $0.12(0.00-7.43)$ & 0.31 \\
\hline \multicolumn{9}{|c|}{ Dual deficiency of the heart and spleen } \\
\hline Lower & $0.66(0.25-1.75)$ & 0.40 & $1.11(0.25-5.06)$ & 0.89 & $0.73(0.16-3.45)$ & 0.69 & $0.69(0.05-9.00)$ & 0.78 \\
\hline Higher & $0.47(0.15-1.53)$ & 0.21 & $0.99(0.18-5.36)$ & 0.99 & $2.24(0.40-12.55)$ & 0.36 & $4.20(0.10-174.71)$ & 0.45 \\
\hline Highest & $0.35(0.08-1.49)$ & 0.16 & $2.81(0.33-24.23)$ & 0.35 & $0.20(0.03-1.26)$ & 0.09 & $5.33(0.08-358.04)$ & 0.44 \\
\hline \multicolumn{9}{|l|}{ Intense heart fire } \\
\hline Lower & $1.33(0.48-3.68)$ & 0.58 & $1.67(0.27-10.49)$ & 0.59 & $0.41(0.09-1.90)$ & 0.25 & $3.34(0.27-41.50)$ & 0.35 \\
\hline Higher & $1.21(0.27-5.34)$ & 0.80 & $0.95(0.06-14.67)$ & 0.97 & $0.14(0.02-1.12)$ & 0.06 & $10.13(0.21-480.14)$ & 0.24 \\
\hline Highest & $0.88(0.19-4.03)$ & 0.87 & $1.30(0.08-21.36)$ & 0.85 & $0.25(0.02-2.72)$ & 0.25 & $0.57(0.01-25.13)$ & 0.77 \\
\hline \multicolumn{9}{|l|}{ Phlegm turbidity } \\
\hline Lower & $0.65(0.30-1.41)$ & 0.27 & $0.52(0.13-2.02)$ & 0.34 & $0.20(0.05-0.75)$ & $<0.05$ & $9.28(0.49-176.75)$ & 0.14 \\
\hline Higher & $2.08(0.67-6.44)$ & 0.21 & $0.31(0.04-2.27)$ & 0.25 & $1.89(0.34-10.43)$ & 0.47 & $11.80(0.37-379.25)$ & 0.16 \\
\hline Highest & $1.35(0.39-4.69)$ & 0.64 & $0.14(0.01-1.61)$ & 0.11 & $0.61(0.09-4.16)$ & 0.61 & $0.96(0.05-19.59)$ & 0.98 \\
\hline \multicolumn{9}{|c|}{ Phlegm fire harassing the heart } \\
\hline Lower & $1.65(0.62-4.40)$ & 0.32 & $0.60(0.14-2.70)$ & 0.51 & $3.93(0.88-17.49)$ & 0.07 & $0.05(0.00-0.76)$ & $<0.05$ \\
\hline Higher & $2.94(0.77-11.25)$ & 0.12 & $3.71(0.37-36.72)$ & 0.26 & $1.60(0.21-12.30)$ & 0.65 & $0.42(0.02-10.61)$ & 0.60 \\
\hline Highest & $1.63(0.39-6.87)$ & 0.50 & $75.83(3.02-1903.66)$ & $<0.01$ & $10.57(0.74-151.16)$ & 0.08 & $0.01(0.00-0.49)$ & $<0.05$ \\
\hline \multicolumn{9}{|c|}{ Liver yin deficiency } \\
\hline Lower & $0.92(0.35-2.46)$ & 0.87 & $0.11(0.02-0.72)$ & $<0.05$ & $2.35(0.50-11.19)$ & 0.28 & $3.71(0.24-57.59)$ & 0.35 \\
\hline Higher & $0.94(0.23-3.82)$ & 0.93 & $0.41(0.03-6.78)$ & 0.54 & $1.72(0.21-13.86)$ & 0.61 & $2.17(0.08-56.47)$ & 0.64 \\
\hline Highest & $0.68(0.16-2.92)$ & 0.60 & $0.10(0.01-1.33)$ & 0.08 & $5.92(0.22-162.83)$ & 0.29 & $12.48(0.14-1124.80)$ & 0.27 \\
\hline \multicolumn{9}{|c|}{ Heart yin deficiency } \\
\hline Lower & $1.09(0.37-3.22)$ & 0.87 & $8.83(1.13-69.16)$ & $<0.05$ & $1.15(0.24-5.39)$ & 0.86 & $12.69(0.89-181.75)$ & 0.06 \\
\hline Higher & $1.66(0.41-6.74)$ & 0.48 & $3.77(0.39-36.14)$ & 0.25 & $1.57(0.21-12.00)$ & 0.66 & $1.32(0.06-27.70)$ & 0.86 \\
\hline Highest & $1.16(0.25-5.51)$ & 0.85 & $12.64(0.70-229.52)$ & 0.09 & $2.31(0.19-27.57)$ & 0.51 & $1.34(0.03-60.17)$ & 0.88 \\
\hline \multicolumn{9}{|c|}{ Kidney yin deficiency } \\
\hline Lower & $1.71(0.67-4.36)$ & 0.26 & $2.70(0.62-11.82)$ & 0.19 & $0.30(0.06-1.46)$ & 0.14 & $1.31(0.13-13.22)$ & 0.82 \\
\hline Higher & $1.54(0.53-4.51)$ & 0.43 & $4.25(0.50-36.21)$ & 0.19 & $0.47(0.08-2.69)$ & 0.40 & $0.12(0.01-2.97)$ & 0.20 \\
\hline Highest & $1.17(0.27-5.07)$ & 0.84 & $0.14(0.01-2.97)$ & 0.20 & $0.20(0.02-2.25)$ & 0.19 & $0.05(0.00-3.07)$ & 0.15 \\
\hline
\end{tabular}

with all forms of anxiety disorders in the younger female group. On the contrary, liver yin deficiency was inversely associated in this group. There was no association between all forms of anxiety disorders and any pattern in the other groups.

\section{Association between Mood Disorders and Visceral Patterns}

Associations between major depression and visceral patterns are shown in table 5 . Phlegm fire harassing the heart was significantly associated with major depression in the older female group. In contrast, this pattern was inversely associated in the older male group.

Associations between all forms of mood disorders and visceral patterns were also investigated and are shown in table 6. The results were similar to those with major depression. Phlegm fire harassing the heart was significantly associated with all forms of mood disorders in the younger male group as well as in the older female group. However, this pattern was inversely associated in the older male group. 
Kondo et al.: Age-Related Changes in Visceral Patterns Associated with Anxiety

Disorders or Depression according to Traditional Chinese Medicine

Table 6. Associations between all mood disorders and visceral patterns

\begin{tabular}{|c|c|c|c|c|c|c|c|c|}
\hline \multirow[t]{2}{*}{ Pattern/extent } & \multicolumn{2}{|c|}{ Younger female group } & \multicolumn{2}{|l|}{ Older female group } & \multicolumn{2}{|l|}{ Younger male group } & \multicolumn{2}{|l|}{ Older male group } \\
\hline & OR $(95 \% \mathrm{CI})$ & $\mathrm{p}$ value & OR $(95 \% \mathrm{CI})$ & $\mathrm{p}$ value & OR $(95 \% \mathrm{CI})$ & $\mathrm{p}$ value & OR $(95 \% \mathrm{CI})$ & $\mathrm{p}$ value \\
\hline \multicolumn{9}{|c|}{ Liver qi depression } \\
\hline Lower & $0.81(0.29-2.23)$ & 0.68 & $0.24(0.05-1.19)$ & 0.08 & $0.72(0.17-3.07)$ & 0.65 & $1.73(0.22-13.40)$ & 0.60 \\
\hline Higher & $0.93(0.31-2.78)$ & 0.90 & $0.09(0.01-0.67)$ & $<0.05$ & $1.31(0.27-6.41)$ & 0.74 & $1.23(0.11-13.34)$ & 0.87 \\
\hline Highest & $1.68(0.44-6.38)$ & 0.45 & $0.17(0.02-1.29)$ & 0.09 & $1.56(0.21-11.68)$ & 0.66 & $3.22(0.29-35.46)$ & 0.34 \\
\hline \multicolumn{9}{|c|}{ Liver fire flaming upward } \\
\hline Lower & $0.62(0.23-1.66)$ & 0.34 & $1.08(0.12-9.79)$ & 0.95 & $1.84(0.38-8.78)$ & 0.45 & $0.81(0.08-8.16)$ & 0.85 \\
\hline Higher & $0.71(0.17-2.95)$ & 0.64 & $0.86(0.06-11.86)$ & 0.91 & $0.65(0.08-5.46)$ & 0.69 & $0.51(0.04-7.40)$ & 0.62 \\
\hline Highest & $0.55(0.13-2.43)$ & 0.43 & $0.42(0.02-8.15)$ & 0.56 & $0.67(0.04-10.95)$ & 0.78 & $0.15(0.01-4.33)$ & 0.27 \\
\hline \multicolumn{9}{|c|}{ Dual deficiency of the heart and spleen } \\
\hline Lower & $0.80(0.30-2.13)$ & 0.66 & $1.48(0.32-6.95)$ & 0.62 & $0.78(0.17-3.65)$ & 0.75 & $0.88(0.09-8.93)$ & 0.91 \\
\hline Higher & $0.53(0.17-1.72)$ & 0.29 & $1.51(0.26-8.60)$ & 0.65 & $1.51(0.28-7.97)$ & 0.63 & $3.03(0.14-64.54)$ & 0.48 \\
\hline Highest & $0.42(0.10-1.80)$ & 0.24 & $5.62(0.58-54.50)$ & 0.14 & $0.17(0.03-1.09)$ & 0.06 & $4.36(0.10-195.40)$ & 0.45 \\
\hline \multicolumn{9}{|l|}{ Intense heart fire } \\
\hline Lower & $1.24(0.44-3.44)$ & 0.68 & $1.13(0.17-7.33)$ & 0.90 & $0.41(0.09-1.90)$ & 0.25 & $4.46(0.43-46.40)$ & 0.21 \\
\hline Higher & $1.19(0.27-5.25)$ & 0.82 & $0.77(0.05-12.24)$ & 0.85 & $0.10(0.01-0.78)$ & $<0.05$ & $5.77(0.22-154.73)$ & 0.30 \\
\hline Highest & $0.86(0.19-3.95)$ & 0.85 & $1.20(0.07-20.14)$ & 0.90 & $0.18(0.02-1.91)$ & 0.15 & $1.16(0.04-35.97)$ & 0.93 \\
\hline \multicolumn{9}{|l|}{ Phlegm turbidity } \\
\hline Lower & $0.53(0.24-1.17)$ & 0.11 & $0.47(0.12-1.85)$ & 0.28 & $0.20(0.05-0.75)$ & $<0.05$ & $5.74(0.65-50.67)$ & 0.12 \\
\hline Higher & $1.99(0.64-6.19)$ & 0.24 & $0.16(0.02-1.27)$ & 0.08 & $1.50(0.27-8.28)$ & 0.64 & $2.37(0.20-28.45)$ & 0.50 \\
\hline Highest & $1.27(0.36-4.47)$ & 0.71 & $0.09(0.01-1.21)$ & 0.07 & $0.37(0.06-2.39)$ & 0.30 & $0.74(0.06-9.50)$ & 0.82 \\
\hline \multicolumn{9}{|c|}{ Phlegm fire harassing the heart } \\
\hline Lower & $1.77(0.66-4.76)$ & 0.26 & $0.65(0.14-3.02)$ & 0.59 & $4.32(0.96-19.39)$ & 0.06 & $0.10(0.01-0.82)$ & $<0.05$ \\
\hline Higher & $3.59(0.93-13.80)$ & 0.06 & $3.73(0.38-36.60)$ & 0.26 & $2.36(0.31-18.14)$ & 0.41 & $0.91(0.06-14.62)$ & 0.94 \\
\hline Highest & $1.93(0.45-8.20)$ & 0.38 & $82.86(3.11-2,206.29)$ & $<0.01$ & $15.38(1.09-216.63)$ & $<0.05$ & $0.04(0.00-1.02)$ & 0.05 \\
\hline \multicolumn{9}{|c|}{ Liver yin deficiency } \\
\hline Lower & $1.01(0.37-2.70)$ & 0.99 & $0.13(0.02-0.80)$ & $<0.05$ & $2.19(0.47-10.31)$ & 0.32 & $4.14(0.57-30.02)$ & 0.16 \\
\hline Higher & $0.94(0.23-3.88)$ & 0.93 & $0.47(0.03-7.63)$ & 0.59 & $2.03(0.26-16.12)$ & 0.50 & $2.83(0.25-32.46)$ & 0.40 \\
\hline Highest & $0.59(0.14-2.57)$ & 0.48 & $0.11(0.01-1.48)$ & 0.10 & $4.58(0.17-122.04)$ & 0.36 & $20.69(0.57-748.45)$ & 0.10 \\
\hline \multicolumn{9}{|c|}{ Heart yin deficiency } \\
\hline Lower & $0.85(0.29-2.53)$ & 0.77 & $5.24(0.66-41.43)$ & 0.12 & $1.21(0.26-5.65)$ & 0.81 & $3.91(0.52-29.53)$ & 0.19 \\
\hline Higher & $1.52(0.37-6.24)$ & 0.56 & $2.11(0.22-20.35)$ & 0.52 & $1.50(0.20-11.09)$ & 0.69 & $0.36(0.03-4.91)$ & 0.44 \\
\hline Highest & $1.02(0.21-4.86)$ & 0.98 & $5.88(0.32-107.88)$ & 0.23 & $3.13(0.27-36.60)$ & 0.36 & $0.21(0.01-5.24)$ & 0.34 \\
\hline \multicolumn{9}{|c|}{ Kidney yin deficiency } \\
\hline Lower & $1.95(0.76-5.03)$ & 0.17 & $3.69(0.80-17.05)$ & 0.09 & $0.30(0.06-1.45)$ & 0.13 & $2.06(0.26-16.23)$ & 0.49 \\
\hline Higher & $1.62(0.55-4.75)$ & 0.38 & $8.48(0.86-83.60)$ & 0.07 & $0.42(0.07-2.36)$ & 0.32 & $0.54(0.05-5.80)$ & 0.61 \\
\hline Highest & $1.40(0.32-6.14)$ & 0.65 & $0.20(0.01-4.22)$ & 0.30 & $0.19(0.02-2.27)$ & 0.19 & $0.19(0.01-6.41)$ & 0.35 \\
\hline
\end{tabular}

\section{Discussion}

One limitation of the present study is that pulse, tongue, and abdominal examinations were not conducted. Since no standard scoring system for traditional Chinese medicine is available, for the present study, the extent of each pattern had to be tentatively based on the assessment of the questionnaires. As the subjects were patients at a tertiary medical care center, the results cannot be generalized. However, the large number of participants with more severe and typical disorders enabled the demonstration of many significant associations between disorders and patterns.

According to a review on acupuncture for anxiety disorders, auricular acupuncture is used in most of the clinical studies [14]. However, somatic acupuncture is considered for particular visceral patterns in some studies [14]. For example, Liu et al. [15] used ST36, PC6, ST25, LR3, BL23, GV4, and LI11 in addition to auricular acupuncture. These meridian points 
Kondo et al.: Age-Related Changes in Visceral Patterns Associated with Anxiety

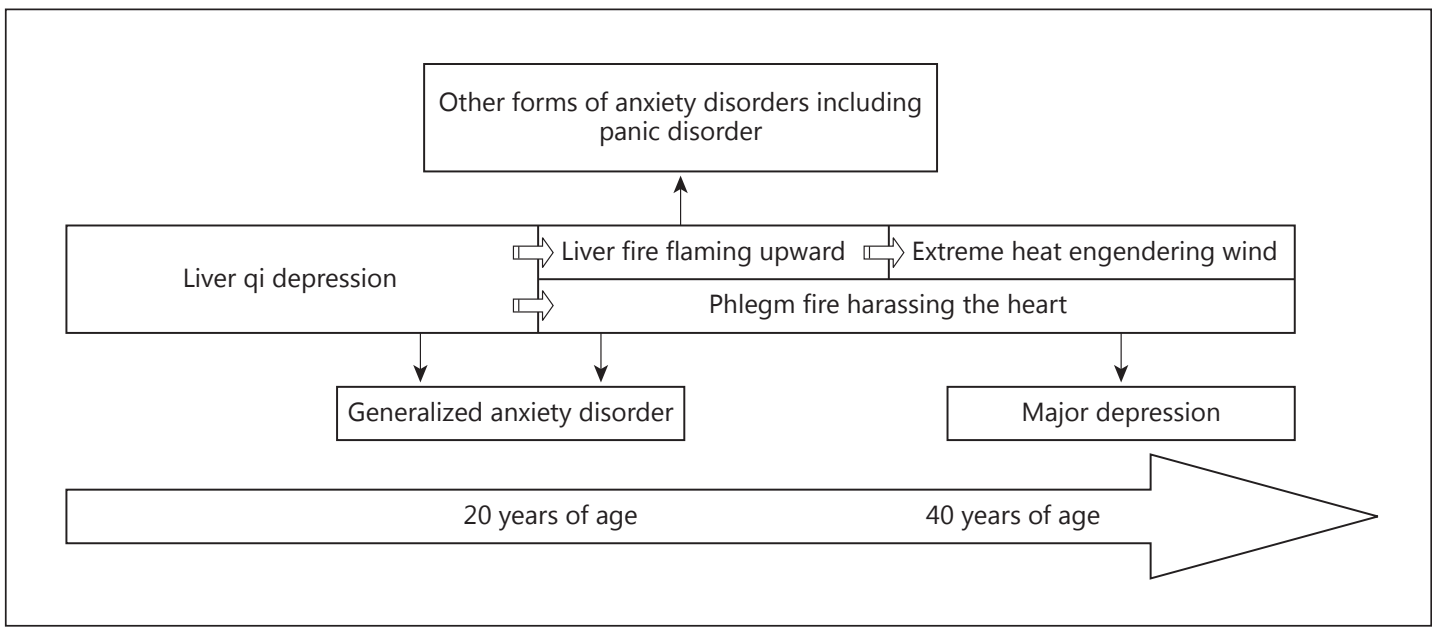

Fig. 1. The central bar indicates the time course of the patterns in traditional Chinese medicine. Liver qi depression sometimes changes to liver fire flaming upward and sometimes to phlegm fire harassing the heart. The thin arrows indicate disorders deriving from each pattern.

consist of those that are effective for liver qi depression (LR3 and PC6), extreme heat engendering wind (LI11), spleen or stomach patterns (ST36, PC6, and ST25) and kidney deficiency (BL23 and GV4). Among these patterns, only liver fire flaming upward was indicated to be associated with anxiety disorders in the present study. However, liver fire flaming upward indicated in the present study results from liver qi depression and leads to extreme heat engendering wind.

Therefore, the point combination used by Liu et al. [15] is partially consistent with the present study. Chao-Ying [16] treated anxiety disorders with Yintang, GV20, GB5, and GB20. Since all meridian points except for Yintang are for liver fire flaming upward and extreme heat engendering wind, which results from liver fire flaming upward, this is consistent with the present study.

Since fear is managed by the kidney, it was expected that the kidney pattern would be associated with all forms of anxiety disorders or generalized anxiety disorder. However, this was not the case. Instead, liver qi depression was significantly associated with generalized anxiety disorder in the younger female group. Although there have been few reports on the patterns associated with generalized anxiety disorder, Zhiling et al. [17] treated it with PC6, HT7, CV17, SP6, etc., which are meridian points for dual deficiency of the heart and spleen. Yuan et al. [18] also used almost the same meridian points (PC6, HT7, SP6, etc.) for generalized anxiety disorder. These reports are inconsistent with the present study. However, the result in the present study is consistent with the fact that elevation in muscle tonus, which is a symptom of liver qi depression, is also included in the criteria for generalized anxiety disorder [9].

In our study, the pattern associated with generalized anxiety disorder was different from the pattern associated with all forms of anxiety disorders. Although no pattern was significantly associated with panic disorder itself, liver fire flaming upward was significantly associated with all forms of anxiety disorders, which includes a large number of subjects with panic disorder. The onset of generalized anxiety disorder is often in the teens. This is comparatively earlier than for other forms of anxiety disorders, especially panic disorder, whose onset is at around 25 years of age and later than for other forms of anxiety disorders [4]. This order is consistent with the traditional Chinese theory that liver fire flaming upward results 
from long-lasting liver qi depression via depletion of yin fluid and appears later than liver qi depression. The schematic progress of patterns and disorders corresponding to the patterns is shown in figure 1. Liver qi depression itself would cause generalized anxiety disorder in the early stage of life. If liver qi depression evolves into liver fire flaming upward, all forms of anxiety disorders, including panic disorder, would occur especially in young females.

Phlegm fire harassing the heart was significantly associated with major depression in the older female group and with all forms of mood disorders in this group and in the younger male group. In previous studies, depression was treated as spleen deficiency [19], dual deficiency of the heart and spleen [19], spleen-kidney yang deficiency [19], liver qi depression [19], and kidney deficiency [20]. Among these reported visceral patterns, the ORs for dual deficiency of the heart and spleen, and liver qi depression were relatively high in the present study, but they were not significantly associated with depression. Zhang et al. [3] treated depression with meridian points including ST40 which resolves phlegm. This is consistent with the association between major depression and phlegm fire harassing the heart in the present study. According to the traditional Chinese theory, liver qi depression evolves into not only liver fire flaming upward but also phlegm fire harassing the heart as shown in figure 1. The time course of these patterns is consistent with the fact that the average onset of major depression is in the late twenties and later than for generalized anxiety disorder. In contrast, phlegm fire harassing the heart was inversely associated with major depression in the older male group. Instead, the ORs of liver yin deficiency associated with major depression and all forms of mood disorders were relatively high in the older male group, although the association was not significant. Older males would tend to suffer from lack of fluid or yin. These results indicate that the patterns attributed to major depression or mood disorders are quite different according to age and sex.

In addition to the result mentioned above, in the present study, no associations between disorders and visceral patterns were indicated in males. This may be attributed to the smaller number of male subjects compared to females.

In summary, age-related changes in the association between the disorders and the patterns were consistent with the age-related progress of both patterns and disorders.

\section{Acknowledgment}

No grants or financial support were received for this study.

\section{Appendix 1}

The Correspondence between Symptoms Described as Chief Complaints or in the KMI and Each Visceral Pattern

\begin{tabular}{|c|c|c|c|c|c|c|c|c|c|c|}
\hline Symptom & $\begin{array}{l}\text { KMI } \\
\text { question } \\
\text { number }\end{array}$ & $\begin{array}{l}\text { Liver qi } \\
\text { depression }\end{array}$ & $\begin{array}{l}\text { Liver fire } \\
\text { flaming } \\
\text { upward }\end{array}$ & $\begin{array}{l}\text { Intense } \\
\text { heart } \\
\text { fire }\end{array}$ & $\begin{array}{l}\text { Phlegm fire } \\
\text { harassing } \\
\text { the heart }\end{array}$ & $\begin{array}{l}\text { Phlegm } \\
\text { turbidity }\end{array}$ & $\begin{array}{l}\text { Dual deficien- } \\
\text { cy of the heart } \\
\text { and spleen }\end{array}$ & $\begin{array}{l}\text { Liver } \\
\text { yin defi- } \\
\text { ciency }\end{array}$ & $\begin{array}{l}\text { Heart } \\
\text { yin defi- } \\
\text { ciency }\end{array}$ & $\begin{array}{l}\text { Kidney } \\
\text { yin defi- } \\
\text { ciency }\end{array}$ \\
\hline Abdominal fullness & & & & & & & O & & & \\
\hline Abdominal wind & & $\mathrm{O}$ & & & & & & & & \\
\hline Abortion & & & & & & & & & & $\mathrm{O}$ \\
\hline Affective lability & & & & & $\mathrm{O}$ & & & & & \\
\hline Afternoon fever & & & & & $\mathrm{O}$ & & & & $\mathrm{O}$ & $\mathrm{O}$ \\
\hline Alopecia & & & & & & & & & & $\mathrm{O}$ \\
\hline Alveolar pyorrhea & & & & & & & & & & $\mathrm{O}$ \\
\hline Amnesia & & & & & & & $\mathrm{O}$ & & $\mathrm{O}$ & \\
\hline
\end{tabular}


Kondo et al.: Age-Related Changes in Visceral Patterns Associated with Anxiety

Disorders or Depression according to Traditional Chinese Medicine

\begin{tabular}{|c|c|c|c|c|c|c|c|c|c|c|}
\hline Symptom & $\begin{array}{l}\text { KMI } \\
\text { question } \\
\text { number }\end{array}$ & $\begin{array}{l}\text { Liver qi } \\
\text { depression }\end{array}$ & $\begin{array}{l}\text { Liver fire } \\
\text { flaming } \\
\text { upward }\end{array}$ & $\begin{array}{l}\text { Intense } \\
\text { heart } \\
\text { fire }\end{array}$ & $\begin{array}{l}\text { Phlegm fire } \\
\text { harassing } \\
\text { the heart }\end{array}$ & $\begin{array}{l}\text { Phlegm } \\
\text { turbidity }\end{array}$ & $\begin{array}{l}\text { Dual deficien- } \\
\text { cy of the heart } \\
\text { and spleen }\end{array}$ & $\begin{array}{l}\text { Liver } \\
\text { yin defi- } \\
\text { ciency }\end{array}$ & $\begin{array}{l}\text { Heart } \\
\text { yin defi- } \\
\text { ciency }\end{array}$ & $\begin{array}{l}\text { Kidney } \\
\text { yin defi- } \\
\text { ciency }\end{array}$ \\
\hline Anorexia & $\mathrm{C}-07$ & & & & 0 & 0 & 0 & & & \\
\hline Anxiety & B-19 & & & & & & O & & & \\
\hline Appendicular numbness & & & & & & & & 0 & & \\
\hline Asthenopia & C-18 & & & & & & & 0 & & \\
\hline Bitter taste in the mouth & & & 0 & & & & & & & \\
\hline Breathlessness & & & & & & & & & 0 & \\
\hline Burping & & 0 & & & & & & & & \\
\hline Cheilitis & & & & 0 & & & & & & \\
\hline Chest oppression & & 0 & & O & & & & & O & \\
\hline Compulsion & & & & & & & & & 0 & \\
\hline Conjunctival hyperemia & & & 0 & & 0 & & & & & \\
\hline Constipation & & $\mathrm{O}$ & O & O & O & & & & & \\
\hline Convulsion & & $\mathrm{O}$ & & & & & & O & & \\
\hline Cramp & & & & & & & & 0 & & \\
\hline Delayed menstruation & & 0 & & & & & $\mathrm{O}$ & 0 & & 0 \\
\hline Delirium & & & & & $\mathrm{O}$ & & & & & \\
\hline Destructive act & & & & & 0 & & & & & \\
\hline Diarrhea & & 0 & & & & & $\mathrm{O}$ & & & \\
\hline Difficulty in falling asleep & C- 44 & & $\mathrm{O}$ & 0 & 0 & & 0 & & 0 & \\
\hline Disruption of sleep & & & $\mathrm{O}$ & $\mathrm{O}$ & 0 & & O & & $\mathrm{O}$ & \\
\hline Dizziness & $\mathrm{C}-38$ & & $\mathrm{O}$ & & & & O & O & O & 0 \\
\hline Early morning awakening & & & 0 & $\mathrm{O}$ & 0 & & O & & $\mathrm{O}$ & \\
\hline Easy fatigability & & & & & & & O & & & \\
\hline Epigastric oppression & & & & & & & 0 & & & \\
\hline Excessive dreaming & $\mathrm{C}-46$ & & 0 & $\mathrm{O}$ & 0 & & O & & $\mathrm{O}$ & \\
\hline Fasciculation & C-43 & O & & & & & & 0 & & \\
\hline $\begin{array}{l}\text { Feeling of heaviness in upper } \\
\text { limbs }\end{array}$ & & $\mathrm{O}$ & & & & & & & & \\
\hline Feeling of thoracic heat & & & & 0 & & & & & & \\
\hline Flush & & & 0 & $\mathrm{O}$ & 0 & & & & $\mathrm{O}$ & 0 \\
\hline Frequent urination & $\mathrm{C}-31$ & 0 & & & & & & & & \\
\hline General fatigue & C-03 & & & & & & O & & & \\
\hline Genital bleeding & & & $\mathrm{O}$ & & & & O & & & \\
\hline Gray hair & & & & & & & & & & 0 \\
\hline Headache & & & $\mathrm{O}$ & & 0 & & & & & \\
\hline Heavy-headed feeling & & & & & & & & 0 & & \\
\hline Hematemesis & & & 0 & 0 & & & & & & \\
\hline Hot flush & & & & & & & & 0 & 0 & 0 \\
\hline Hypacusis & & & 0 & & & & & & & 0 \\
\hline Hyperkinesia & & & & & 0 & & & & & \\
\hline Hypersexuality & & & & & & & & & & $\mathrm{O}$ \\
\hline Hyperventilation & & & & & 0 & & & & & \\
\hline Hypobulia & B-43 & & & & & & O & & & \\
\hline $\begin{array}{l}\text { Hyposthenia in the lumbar part } \\
\text { or lower limbs }\end{array}$ & & & & & & & & & & 0 \\
\hline Impatience & B-02 & & & & & & & & 0 & \\
\hline Infertility & & & & & & & & & & 0 \\
\hline Irascibility & & $\mathrm{O}$ & $\mathrm{O}$ & & $\mathrm{O}$ & & & & & \\
\hline Irritation & $\begin{array}{l}\text { B-13 } \\
\text { or } 22\end{array}$ & 0 & O & $\mathrm{O}$ & 0 & $\mathrm{O}$ & & $\mathrm{O}$ & & \\
\hline $\begin{array}{l}\text { Lack of consciousness of } \\
\text { disease }\end{array}$ & & & & & 0 & & & & & \\
\hline Melancholy & B-42 & 0 & & & & 0 & & & & 0 \\
\hline Menorrhargia & & 0 & & & & & 0 & & & \\
\hline Menorrhargia & & & 0 & & & & $\mathrm{O}$ & & & \\
\hline Morning fever & & & & & 0 & & & & & \\
\hline Myalgia & & & & & & & & 0 & & \\
\hline Nasal hemorrhage & & & & 0 & & & & & & \\
\hline Nausea & & $\mathrm{O}$ & & & & & 0 & & & \\
\hline Nervosity & $\mathrm{A}-01$ & 0 & & & & & & & & 0 \\
\hline Night sweating & & & & & & & & 0 & 0 & 0 \\
\hline
\end{tabular}


Kondo et al.: Age-Related Changes in Visceral Patterns Associated with Anxiety

Disorders or Depression according to Traditional Chinese Medicine

\begin{tabular}{|c|c|c|c|c|c|c|c|c|c|c|}
\hline Symptom & $\begin{array}{l}\text { KMI } \\
\text { question } \\
\text { number }\end{array}$ & $\begin{array}{l}\text { Liver qi } \\
\text { depression }\end{array}$ & $\begin{array}{l}\text { Liver fire } \\
\text { flaming } \\
\text { upward }\end{array}$ & $\begin{array}{l}\text { Intense } \\
\text { heart } \\
\text { fire }\end{array}$ & $\begin{array}{l}\text { Phlegm fire } \\
\text { harassing } \\
\text { the heart }\end{array}$ & $\begin{array}{l}\text { Phlegm } \\
\text { turbidity }\end{array}$ & $\begin{array}{l}\text { Dual deficien- } \\
\text { cy of the heart } \\
\text { and spleen }\end{array}$ & $\begin{array}{l}\text { Liver } \\
\text { yin defi- } \\
\text { ciency }\end{array}$ & $\begin{array}{l}\text { Heart } \\
\text { yin defi- } \\
\text { ciency }\end{array}$ & $\begin{array}{l}\text { Kidney } \\
\text { yin defi- } \\
\text { ciency }\end{array}$ \\
\hline Nightmares & & & & & 0 & & 0 & & & \\
\hline Ophthalmalgia & & & $\mathrm{O}$ & & & & & & & \\
\hline Palpitation & C-09 & & & & & & $\mathrm{O}$ & & 0 & \\
\hline Phobia & & & & & & & & & & 0 \\
\hline Pollution & & & & & & & & & & 0 \\
\hline Premature menstruation & & & 0 & & & & 0 & & & \\
\hline Protracted urination & & $\mathrm{O}$ & & & & & & & & \\
\hline Retardation of thought & C-36 & & & & & & & & 0 & \\
\hline Rugitus & & 0 & & & & & & & & \\
\hline Sadness & & & & & $\mathrm{O}$ & & & & & \\
\hline Sense of residual urine & & 0 & & & & & & & & \\
\hline Sexless & & 0 & & & & & & & & \\
\hline$\overline{\text { Sigh }}$ & & $\mathrm{O}$ & & & & & & & & \\
\hline Slight menorrhea & & & & & & & & 0 & & 0 \\
\hline Sporadic headache & & & $\mathrm{O}$ & & & & & & & \\
\hline Sputum & & & & & $\mathrm{O}$ & & & & & \\
\hline Stiffness in shoulders & & 0 & & & & & & & & \\
\hline Stomatitis & & & & 0 & 0 & & & & & \\
\hline Systemic convulsion & C-41 & 0 & & & & & & 0 & & \\
\hline Thirst & $\mathrm{C}-20$ & & 0 & 0 & $\mathrm{O}$ & & & $\mathrm{O}$ & $\mathrm{O}$ & $\mathrm{O}$ \\
\hline Tinnitus & C-19 & & 0 & & & & & & & 0 \\
\hline $\begin{array}{l}\text { Unpleasant sensation of } \\
\text { laryngopharynx }\end{array}$ & & $\mathrm{O}$ & & & & & & & & \\
\hline $\begin{array}{l}\text { Vexing heat in the chest, } \\
\text { palms and soles }\end{array}$ & & & & & & & & & 0 & 0 \\
\hline Vomiting & & O & & & & & 0 & & & \\
\hline Weight loss & C-08 & & & & & & & $\mathrm{O}$ & $\mathrm{O}$ & $\mathrm{O}$ \\
\hline Xerasia & & & & & & & & & & 0 \\
\hline Xeroderma & & & & & & & & 0 & 0 & 0 \\
\hline
\end{tabular}

\section{References}

1 Warden D, Rush AJ, Trivedi MH, Fava M, Wisniewski SR: The STAR D Project results: a comprehensive review of findings. Curr Psychiatry Rep 2007;9:449-459.

2 Eisenberg L: Complementary and alternative medicine: what is its role? Harv Rev Psychiatry 2002;10:221230.

3 Zhang GJ, Shi ZY, Liu S, Gong SH, Liu JQ, Liu JS: Clinical observation on treatment of depression by electroacupuncture combined with Paroxetine. Chin J Integr Med 2007;13:228-230.

4 Kaiya H: Anxious depression (Fuan-yokuutsu syoukougun). Bunshi Seishin Igaku 2013;13:84-88.

5 Mukai M, Baba S, Asao T, Suzuki F, Aoyama H: Traditional Chinese medicine in psychiatry: liver diseases and blood-stagnation (in Japanese). Clin J Trad Chin Med 2002;24:88-90.

6 Mukai M, Baba S, Suzuki F: Traditional Chinese medicine in psychiatry: intense heart fire pattern (in Japanese). Clin J Trad Chin Med 2002;23:70-73.

7 Mukai M, Baba S, Asao T, Suzuki F, Aoyama H, Fujii K: Traditional Chinese medicine in psychiatry: deficiency pattern (in Japanese). Clin J Trad Chin Med 2002;24:78-80.

8 Mukai M, Baba S, Suzuki F: Traditional Chinese medicine in psychiatry: pattern of phlegm-fire harassing the heart and phlegm turbidity (in Japanese). Clin J Trad Chin Med 2002;23:72-75.

9 American Psychiatric Association: DSM-IV: Diagnostic and Statistical Manual of Mental Disorders, ed 4. Washington, American Psychiatric Association, 1994.

10 Matsuoka Y: KMI (in Japanese); in Kawano T, Suematsu H, Arazato R (eds): Psychological Test for Psychosomatic Medicine. Tokyo, Asakura Publications, 1990, pp 45-49.

11 WHO Regional Office for the Western Pacific: WHO International Standard Terminologies on Traditional Medicine in the Western Pacific Region. Manila, WPRO Nonserial Publication, 2007.

12 Nakasato K: Evaluation of depressive state (Yokuutsu hyouteihou); in Kono T, Suematsu H, Shinzato R (eds): Shinshin igaku no tame no shinri Test. Tokyo, Asakura Publications, 1990, pp 36-39.

13 Nakasato K: STAI; in Kono T, Suematsu H, Shinzato R: Shinshin igaku no tame no shinri Test. Tokyo, Asakura Publications, 1990, pp 27-31. 
14 Pilkington K, Kirkwood G, Rampes H, Cummings M, Richardson J: Acupuncture for anxiety and anxiety disorders - a systematic literature review. Acupunct Med 2007;25:1-10.

15 Liu G, Zang Y, Guo L, Liu A: Comparative study on acupuncture combined with behavioural desensitisation for treatment of anxiety neurosis. Am J Acupunct 1998;26:117-120.

16 Chao-Ying W: The electroacupuncture treatment of 20 cases of anxiety disorder. Hunan Journal of Traditional Chinese Medicine 2003;3:26.

17 Zhiling W, Yuhong L, Hong L: Acupuncture treatment of generalized anxiety disorder. J Tradit Chin Med 2006; 26:170-171.

18 Yuan Q, Li JN, Liu B, Wu ZF, Jin R: Effect of Jin-3-needling therapy on plasma corticosteroid, adrenocorticotrophic hormone and platelet 5-HT levels in patients with generalized anxiety disorder. Chin J Integr Med 2007;13:264-268.

19 Wang XJ, Wang LL, Qiao HF, Li JB: Observation on effective characteristics of acupuncture combined with medicine on depression of different syndrome types (in Chinese). Zhongguo Zhen Jiu 2009;29:699-702.

20 Wang YJ, Shi Q, Jiang JC, Liang QQ, Bian Q, Li CG, et al: Establishment of a rat model of cervical syndrome with qi deficiency, blood stasis and kidney deficiency (in Chinese). Zhong Xi Yi Jie He Xue Bao 2008;6:1152-1158. 